\title{
Role of Colombo Medical Faculty in the development of research: the past, present and the future
}

\author{
Chandrika Wijeyaratne \\ University of Colombo, Sri Lanka
}

The Faculty of Medicine, University of Colombo, over its remarkable journey of 150 years, has left a legacy of research par excellence. The institution at present enjoys a vibrant research ethos and a deep research commitment, with time-appropriate research outputs and outcomes. This makes us extremely proud and humbled. The current teachers and students of the faculty are strengthened in looking to the future with confidence with a deep commitment to upscale their research program. Reflecting upon the birth and sustenance of this magnificent heritage and its long journey over the differing eras of medical education and research settings, we salute our pioneers. Successive generations of academics have had a clear vision to encourage a sustained faculty-based commitment to research that is ably interwoven with teaching-learning and service. We have no doubt, that the quest for determining the truth through research was the sole purpose then and now. Sharing and dissemination of results of research and their application to clinical practice, are being carried out for the greater good of humanity.

We are well aware that the initial focus on medical research was anecdotal and chiefly clinical, predominantly on tropical diseases and envenomation, with case reports and discussions on proper diagnosis and appropriate treatment. Epidemiological evidence was viewed as a vital piece of information in health policy and planning

Author responsible for correspondence:

Chandrika Wijeyaratne

Vice Chancellor, University of Colombo

Senior Professor in Reproductive Medicine

Faculty of Medicine,

University of Colombo

Email: chandrika@obg.cmb.ac.lk

https://orcid.org/0000-0002-4563-5662

DOI: http://doi.org/10.4038/cjms.v56i1.4962 cardiovascular risks and other common noncommunicable diseases have contributed towards international guideline development through systematic reviews. With the advent of improvement in laboratory and genetic diagnostics by the mid 1980s, the molecular and cellular basis of medicine became an essential component of the research arena. Our faculty did not lose out on this and ventured into multidisciplinary and regional / international multicenter studies. Such research in the basic sciences, particularly in molecular biology, are well cited in the literature. Concurrently, commendable achievements of Sri Lanka in maternal and child health, with excellent outcomes in the immunization programme, establishing the scientific basis of the value of exclusive breast feeding and the disease potential of cow's milk, while addressing malnutrition and diarrhea, were well supported by researchers in our faculty, public health experts and clinicians alike, having a strong social outlook. The names of these individual academic leads need to be etched in our memory books with gratitude and reverence. The emergence of the pharmaceutical industry was studied scientifically by our academics. The objective analysis of an evidencebased approach to the rational prescription of medications has helped the world at large to take a pragmatic approach to generic drug prescriptions. The advent of solid organ transplantation and their successes have also been reported for the first time in Sri Lanka by this same faculty, enabling the victims and families of end-organ failure to be infused with new life. More recently, the scientific basis of health promotion and behavioural change communication on the prevention and control of lifestyle diseases has been recognized by groups within our faculty, as well as the relationship between mind and body in health and disease through mindfulness meditation. These are only a few examples of a large repository.

The need to inculcate a questioning mind and an 
analytical thought process from medical student days was recognized at the turn of the millennium. This paved the way for a sustained student research participation and training. Approximately one-third of our academics are directly involved in supervising research degrees. In parallel, we have witnessed a substantial expansion of the publication numbers and $\mathrm{h}$ indices among the research community. The University of Colombo would greatly appreciate a cohort of like-minded, committed scientific personnel with an excellent track record of research with honesty and integrity to give leadership to research management with a duality of purpose. While research prioritization, training in research methodology and data analysis alongside a sustained research funding through multiple sources must be given due attention; the formulation of a digital platform to collate all research reports and encourage publishing must be addressed. Meanwhile our University's current quest to upscale research outcomes through technology transfer and commercialization must be borne in mind. With respect to the dissemination of research from the very inception of our faculty, the written form of communication was the only available medium for learning through the sharing of objective findings. The fact that the Ceylon Medical Journal (CMJ), then termed the journal of the Ceylon Branch of the British Medical Association, commenced publication in 1887, just a few years after the establishment of the Colombo Medical School, clearly confirms the vision and commitment of our founders to inculcate an inquiring mind among learners and practitioners alike. There is no doubt that our forefathers identified focused research and the dissemination of research findings as an essential component of a vibrant academic environment. The Ceylon Journal of Medical Science (CJMS), the first official publication of the Faculty of Medicine, shall commemorate with pride the $150^{\text {th }}$ anniversary of its parent institution. 